\title{
Criminologie
}

\section{Les professionnels de la surveillance financière. Le malentendu comme condition de possibilité}

\section{Anthony Amicelle}

Volume 46, numéro 2, automne 2013

Nouveaux regards sur les métiers de la sécurité

URI : https://id.erudit.org/iderudit/1020993ar

DOI : https://doi.org/10.7202/1020993ar

Aller au sommaire du numéro

\section{Éditeur(s)}

Les Presses de l’Université de Montréal

ISSN

0316-0041 (imprimé)

1492-1367 (numérique)

Découvrir la revue

Citer cet article

Amicelle, A. (2013). Les professionnels de la surveillance financière. Le malentendu comme condition de possibilité. Criminologie, 46(2), 195-219. https://doi.org/10.7202/1020993ar
Résumé de l'article

Le suivi de la piste financière fait aujourd'hui partie des pratiques instituées en matière de police et de renseignement. Située à l'interface de la finance et de la sécurité, la lutte contre le blanchiment de capitaux et le financement du terrorisme repose sur les relations de coopération tissées entre deux groupes d'acteurs aux motivations et aux savoir-faire différents. La notion floue d'« argent sale » est en effet le point de rencontre d'une constellation d'intérêts et de deux logiques d'action propres aux forces de l'ordre et aux opérateurs du système financier. Analysant le dispositif anti-blanchiment au Royaume-Uni, le présent article met en lumière l'articulation fragile de ces deux logiques d'action autour d'un malentendu sur le sens donné à la lutte contre l'« argent sale ». Loin de constituer un obstacle à l'élaboration et à l'opérationnalisation d'une politique spécifique, le malentendu est ici sa condition même de possibilité. Méprises et divergences d'interprétation sur le " problème » à traiter et les objectifs à poursuivre contribuent paradoxalement à la formation du bataillon bigarré des professionnels de la surveillance financière. 


\title{
Les professionnels de la surveillance financière. \\ Le malentendu comme condition de possibilité ${ }^{1}$
}

\author{
Anthony Amicelle ${ }^{2}$ \\ Professeur adjoint \\ École de criminologie, Université de Montréal \\ Chercheur régulier \\ Centre international de criminologie comparée (CICC) \\ anthony.amicelle@umontreal.ca
}

\begin{abstract}
RÉSUMÉ - Le suivi de la piste financière fait aujourd'hui partie des pratiques instituées en matière de police et de renseignement. Située à l'interface de la finance et de la sécurité, la lutte contre le blanchiment de capitaux et le financement du terrorisme repose sur les relations de coopération tissées entre deux groupes d'acteurs aux motivations et aux savoir-faire différents. La notion floue $d^{\prime}$ ' argent sale» est en effet le point de rencontre d'une constellation d'intérêts et de deux logiques d'action propres aux forces de l'ordre et aux opérateurs du système financier. Analysant le dispositif anti-blanchiment au Royaume-Uni, le présent article met en lumière l'articulation fragile de ces deux logiques d'action autour d'un malentendu sur le sens donné à la lutte contre l'«argent sale». Loin de constituer un obstacle à l'élaboration et à l'opérationnalisation d'une politique spécifique, le malentendu est ici sa condition même de possibilité. Méprises et divergences d'interprétation sur le «problème» à traiter et les objectifs à poursuivre contribuent paradoxalement à la formation du bataillon bigarré des professionnels de la surveillance financière.
\end{abstract}

MOTS CLÉS • argent sale, malentendu, renseignement, risque, surveillance.

1. L'auteur remercie les évaluateurs anonymes pour leurs précieux commentaires.

2. Anthony Amicelle, Université de Montréal, Pavillon Lionel-Groulx, École de criminologie, C.P. 6128, succursale Centre-Ville, Montréal, (Québec) CANADA, H3C 3J7

Criminologie, vol. $46, \mathrm{n}^{\circ} 2$ (2013) 
On ne sait jamais, sur un malentendu ça peut marcher... Jean-Claude Dusse

Le monde ne marche que par le malentendu. C'est par le malentendu que tout le monde s'accorde. Car si, par malheur, on se comprenait, on ne pourrait jamais s'accorder.

Charles-Pierre Baudelaire

\section{Introduction}

La lutte contre l'«argent sale» recouvre un champ d'illégalismes extrêmement étendu. Ce dispositif cible ainsi les agissements ayant pour but de déguiser l'origine de capitaux issus d'activités illégales. L'usage de fonds provenant d'activités aussi bien légales qu'illégales à des fins de financement du terrorisme est également visé. Enfin, le dispositif porte de plus en plus sur les pratiques de dissimulation de l'existence ou de la nature de revenus obtenus légalement à l'instar de nombreuses pratiques de blanchiment de fraude fiscale (Amicelle, 2013).

Le suivi de la piste financière fait désormais partie des pratiques instituées au sein des forces de l'ordre. L'importance donnée à la surveillance financière peut être résumée sous forme d'une expression circulant dans le champ de la sécurité. Alors que l'empereur romain Vespasien avait décrété en son temps que l'argent n'avait pas d'odeur (pecunia non olet) et que Gordon Gekko affirmait que l'argent ne dormait jamais (money never sleeps), nombre d'agents de police et de renseignement considèrent pour leur part que l'argent ne ment pas (money does not lie) (Levi, 2010; Amicelle, 2012; De Goede, 2012). La fiabilité supposée du renseignement financier est régulièrement mise en avant. Ce postulat est particulièrement prégnant dans les discours de légitimation des multiples mesures prônées contre le financement du terrorisme au cours des années 2000.

«Suivre l'argent» est une des sources d'information les plus précieuses que nous ayons pour identifier et localiser les réseaux de terroristes et leurs soutiens. Si un associé terroriste que nous sommes en train de surveiller envoie ou reçoit de l'argent d'une autre personne, nous savons qu'il y a un lien entre les deux individus. Et, alors que les soutiens des terroristes peuvent utiliser des noms de code au téléphone, quand ils envoient ou reçoivent de l'argent via le système bancaire, ils fournissent souvent des informations qui donnent le type de pistes concrètes pouvant faire avancer une enquête. Pour ces raisons, les agents du contreterrorisme placent beaucoup d'espoir dans le renseignement financier ${ }^{3}$. (Levey, 2006)

3. Au moment de cette déclaration, Stuart Levey occupait les fonctions de soussecrétaire d'État sur le terrorisme et le renseignement financier aux États-Unis. 
La priorité donnée à la lutte contre le financement du terrorisme a d'ailleurs accentué et modifié sensiblement l'attention portée à la surveillance et au renseignement financier en l'associant à une logique préemptive. La piste financière n'est plus seulement promue pour identifier et confisquer les produits d'un crime d'ores et déjà commis, elle l'est aussi pour empêcher la survenue éventuelle d'attentats. Ainsi, la lutte contre l'«argent sale» vise autant à produire des preuves permettant de poursuivre et de condamner des criminels qu'à amasser des renseignements confidentiels afin de mettre en incapacité des suspects de terrorisme avant qu'ils n'aient agi ${ }^{4}$. Modèle du genre en Europe, la stratégie britannique attribue un rôle multiple au renseignement financier, que ce soit pour faciliter les enquêtes sur un crime ou un attentat (look backwards), pour cartographier les associations de malfaiteurs en visualisant les relations financières d'un suspect (look sideways) ou encore pour identifier les signes avant-coureurs d'une activité criminelle ou terroriste en préparation (look forward) (HM Treasury, 2007).

Enfin, qu'il soit associé à des velléités répressives, dissuasives, préventives ou préemptives, et mobilisé contre des actes de financement $\mathrm{du}$ terrorisme ou de blanchiment d'argent, le renseignement financier a surtout pour spécificité de résulter des relations de coopération tissées entre forces de l'ordre et opérateurs du système financier. Les données collectées et analysées par les cellules nationales de renseignement financier proviennent essentiellement d'institutions financières ayant pour obligation légale de les informer lorsqu'elles «savent, soupçonnent ou ont de bonnes raisons de soupçonner» la présence d'«argent sale» (Union européenne, 2005). Les représentants de ces institutions doivent faire preuve de proactivité en dénonçant de leur propre initiative leurs clients mais aussi de réactivité en répondant aux demandes d'informations sollicitées par les agents des cellules de renseignement financier ou de toute autre autorité étatique habilitée.

La routinisation des interactions professionnelles entre les milieux bancaire et policier constitue l'un des principaux effets de deux décennies de mobilisation globale contre l'argent sale. Inimaginables en France à la fin des années 1980, les contacts entre ces deux univers professionnels sont aujourd'hui banalisés. Désormais assumé, l'entretien de relations avec la

4. Pour un questionnement critique de la logique préemptive dans le cadre des pratiques anti-terroristes, voir Bigo, 2006; Vlcek, 2006; De Goede, 2008a; Ericson, 2008; McCullogh \& Pickering, 2009; Hayes \& Sullivan, 2010. 
police ne constitue plus une dimension secrète, voire honteuse, de l'activité bancaire (Favarel-Garrigues et al., 2009: 281).

Bien que les compagnies d'assurances, les sociétés d'audit et les cabinets d'avocats et de notaires soient également soumis aux exigences de vigilance et aux obligations déclaratives, les établissements bancaires et financiers demeurent les premiers pourvoyeurs de signalements, en France comme ailleurs (Commission européenne, 2008; Traitement du Renseignement et Action Contre les Circuits Financiers Clandestins [Tracfin], 2012; Serious Organised Crime Agency [Soca], 2012a).

La lutte contre l'«argent sale» est située à l'interface de la finance et de la sécurité. D'origine informatique, cette notion d'interface est particulièrement pertinente dans le cas présent puisqu'elle désigne les «zones de contact et d'interaction entre deux systèmes à orientation différente» (Lascoumes, 1986: 59). L'objectif de notre article est précisément de rendre compte des pratiques émanant des zones de contact entre les acteurs de la finance et de la sécurité qui forment ensemble le bataillon bigarré des professionnels de la surveillance financière. Banalisée sous différentes formes dans la plupart des États membres de l'Union européenne, l'existence de ces zones de contact a atteint un degré de formalisation inédit au Royaume-Uni. Composés de représentants des grandes banques et d'agents des services de police et de renseignement, de véritables groupes de travail hybrides ont été mis sur pied pour lutter contre l'«argent sale».

Dans quelle mesure des acteurs a priori porteurs de dispositions et d'intérêts si éloignés parviennent-ils à s'accorder pour se mobiliser conjointement contre un «problème public» spécifique? Via l'étude du monde de la surveillance financière au Royaume-Uni, nous souhaitons mettre en lumière la force du malentendu comme condition de possibilité d'un projet politique et d'une action publique. Alors que le malentendu est généralement présenté comme une source de blocage et d'impasse, l'interprétation que nous proposons dans cet article tend au contraire à insister sur sa productivité paradoxale. La divergence d'interprétation d'un «problème public» ou encore la méprise sur le sens d'un mot-clé ont ici un rôle d'entraînement aux effets controversés.

Pour ce faire, nous allons d'abord décrire et analyser la méfiance et les tensions permanentes qui caractérisent la relation de dépendance informationnelle entre professionnels de la surveillance financière. Ensuite, nous donnerons à voir le mode de coopération plus tacite qu'explicite entre 
acteurs «finance» et acteurs «sécurité5». Celui-ci repose sur un malentendu opératoire autour des objectifs concrets de la lutte contre l'«argent sale $^{6} »$. Au Royaume-Uni, ce malentendu est aujourd'hui cristallisé autour de la lingua franca de l'industrie financière et des politiques publiques, celle de la «gestion des risques» (Hood et al., 2001; Power, 2004).

Les éléments empiriques de cet article sont principalement tirés de la soixantaine d'entretiens semi-directifs menés entre 2006 et 2010 auprès d'acteurs européens mobilisés contre l'«argent sale»; notamment à Bruxelles au sein des institutions de l'Union européenne (Conseil, Commission et Parlement) et à Londres au sein des ministères, des services de sécurité et des établissements commerciaux (essentiellement banques de détail et d'investissement, de taille variable, britanniques et étrangères) impliqués dans le dispositif britannique de surveillance financière. D'environ 90 minutes, ces entretiens nous ont fourni un surcroît d'informations factuelles sur la manière dont s'élaborent et s'organisent les pratiques de surveillance financière afin de mieux identifier «qui fait quoi, avec qui et au moyen de quels instruments». Ils nous ont aussi permis de saisir les grilles de lecture et la multiplicité des logiques d'action à partir desquelles la lutte contre l'«argent sale» est appréhendée. La juxtaposition de ces représentations a été in fine d'une grande utilité pour se départir de toute vision consensuelle et mieux cerner les points de tension et les rapports de force structurant le champ de l'antiblanchiment et de la lutte contre le financement du terrorisme ${ }^{7}$.

\section{Des relations finance-sécurité sous tension}

\section{Le modèle britannique de surveillance financière}

Les Britanniques sont les plus avancés. Apparemment, ils ont créé des groupes de travail et ils ont autorisé certaines personnes du secteur finan-

5. Ne prétendant pas à l'exhaustivité en ce qui concerne les acteurs et institutions engagés dans la surveillance financière, la présente analyse porte essentiellement sur les relations entre les agents de la cellule britannique de renseignement financier et ceux d'établissements bancaires et de compagnies d'assurances. De la même façon, l'article porte exclusivement sur les circuits financiers officiels et n'aborde pas la problématique des systèmes de remise de fonds informels souvent résumée de manière hâtive et réductrice au système Hawala. Sur cette problématique, voir notamment Munzele et al., 2008; Haggerty \& Razavy, 2009.

6. L'expression de «malentendu opératoire» est ici reprise du travail de Béatrice Hibou (2011).

7. Sur l'utilisation des entretiens à cet effet, voir Dezalay et Rask Madsen (2006). 
cier à profiter d'une habilitation et à avoir accès à des renseignements confidentiels. Cela leur permet d'avoir un système de surveillance beaucoup plus pointu. C'est bête, mais si vous n'avez pas l'information, vous ne pouvez pas l'utiliser, donc vous cherchez n'importe où. (Entretien avec un fonctionnaire de la direction générale «Marché intérieur» de la Commission européenne, 2008)

Au sein des institutions européennes, le Royaume-Uni est régulièrement érigé en modèle à suivre sur le plan opérationnel avec la routinisation des espaces d'échanges d'informations entre les agents de police et de renseignement et ceux des établissements bancaires et des compagnies d'assurance.

D'un côté, il y a bien évidemment la cellule britannique de renseignement financier qui est une composante de l'Agence nationale contre le crime organisé (Serious Organized Crime Agency - Soca). Hormis les agents de la Soca affectés à la cellule de renseignement financier, la sphère du renseignement et de la police d'État est notamment représentée par l'unité d'investigation financière contre le terrorisme (National Terrorist Financial Investigation Unit). Rattachée à la Metropolitan Police du Grand Londres, cette unité spéciale de Scotland Yard dispose d'une compétence nationale sur les affaires de financement du terrorisme. Enfin, les services de sécurité du Royaume-Uni et les dizaines de forces de police locales présentes sur le territoire ont également des agents habilités en matière de renseignement financier. Près de 3500 individus possèdent une accréditation leur permettant d'accéder au fichier central de la Soca qui regroupe les déclarations de soupçon transmises par les institutions soumises à la législation anti-blanchiment. Ces signalements sont officiellement utilisés pour tous types d'activités criminelles, « de la fraude aux prestations sociales au trafic de drogue international, du trafic d'êtres humains au financement du terrorisme» (Soca, 2012b).

De l'autre côté, au sein des institutions bancaires et financières, les agents de l'État disposent d'interlocuteurs privilégiés en la personne des «agents de signalement du blanchiment d'argent» (Money Laundering Reporting Officers - Mlros). En effet, les dirigeants de ces établissements ont l'obligation légale de nommer un employé chargé de superviser leur dispositif interne de surveillance financière et de signaler tout soupçon ou toute information pertinente aux services compétents, à commencer par la Soca.

Quand j'ai commencé en 1994, il s'agissait d'une fonction dont personne ne voulait vraiment, peu considérée, voire méprisée par les senior managers 
et refilée au personnel subalterne en complément d'autres tâches. Aujourd'hui c'est un métier à part entière, recherché par beaucoup de monde et occupé par des senior managers. Au milieu des années 1990, j'étais le premier Mlro que nous avions, il n'y avait que moi pour la compagnie. Aujourd'hui, l'organisation compte une cinquantaine de Mlros et si l'on ajoute leurs collaborateurs, on arrive à un total de 150 personnes travaillant sur les enjeux de délinquance financière au sein de la compagnie, soit une évolution considérable en une dizaine d'années. (Entretien avec un Mlro, 2008)

La fonction d'agent de conformité anti-blanchiment a réellement émergé dans le sillage de la réglementation nationale de 1993, venant transposer la première directive européenne de 1991 (Union européenne, 1991). D'abord marginale et peu attractive professionnellement, cette fonction est désormais valorisée et constitue un rouage important dans l'organisation interne des institutions mises en première ligne de la surveillance financière (Favarel-Garrigues et al., 2008).

\section{Entre dépendance informationnelle et exigences de confidentialité}

La règle générale a toujours été de dire qu'avec le blanchiment d'argent nous cherchions une aiguille dans une botte de foin, mais avec le financement du terrorisme nous nous sommes mis à chercher une aiguille dans un tas d'aiguilles dans la mesure où il s'agit souvent de transactions banales, or nous gérons des millions de transactions et il nous faudrait connaître l'intention des individus au moment où ils font ces transactions pour qu'elles passent de banales à suspectes, mais sans information préalable des services de sécurité, cela nous est impossible, nous ne savons pas par où commencer $^{8}$.(Entretien avec un Mlro, 2008)

Ce constat critique est partagé avec plus ou moins de nuances par l'ensemble des professionnels de la surveillance financière au point d'être relayé par les auteurs de la stratégie européenne contre le financement du terrorisme (Conseil de l'Union européenne, 2008). Afin de remédier aux difficultés de détection des «finances terroristes», le renforcement des mécanismes d'échange de données entre les acteurs financiers et les acteurs de la sécurité a été élevé au rang de priorité absolue.

8. Sur les spécificités et les difficultés de détection du financement du terrorisme, voir Dupuis-Danon, 2004; Fines, 2004; Amicelle, 2008. 
Bien qu'ils soient en situation de dépendance informationnelle, ces deux groupes d'acteurs ont toujours exprimé des réticences à l'idée de partager les informations en leur possession. D'un côté, les acteurs financiers restent attachés au secret professionnel malgré la levée partielle du principe de non-divulgation des informations relatives à leurs clients. S'ils se disent soucieux de remplir leurs obligations déclaratives, ils se font aussi les gardiens sourcilleux de la confidentialité des affaires financières lorsqu'ils considèrent que leurs interlocuteurs sortent du cadre légal des dérogations anti-blanchiment.

La plupart du temps, nous recevons un coup de téléphone en amont de la production d'une ordonnance du tribunal. Ils nous disent qu'ils sont intéressés par telle personne et nous demandent si cela vaut le coup de lancer la procédure pour obtenir une ordonnance. Nous jetons un œil à nos dossiers et nous leur répondons si oui ou non il y a des choses qu'ils pourraient trouver utiles mais sans leur donner d'informations précises et c'est à partir de cet échange informel qu'ils vont chercher une ordonnance. Quelqu'un d'une agence de sécurité m'a téléphoné ce matin pour des informations sur un client $\mathrm{X}$ et les circonstances étaient $\mathrm{Y}$. Il n'avait pas d'ordonnance du tribunal, mais j'ai considéré que $Y$ était suffisamment sérieux ou urgent pour l'aider. En revanche, si un policier me dit qu'il est intéressé par $\mathrm{X}$ et que je ne suis pas convaincu par $\mathrm{Y}$, dans ce cas-là je ne fais rien. Je lui demande qu'il m'en dise plus et qu'il revienne avec une requête formelle. Le danger est que la demande d'information soit disproportionnée. Dans ces cas-là, nous répondons souvent que oui nous avons l'information mais que nous ne devrions pas la leur fournir sans autorisation préalable car ils vont porter atteinte à la confidentialité des affaires de nos clients et que cet abus nous met dans une position inconfortable. Dans ce genre de situation informelle, ils ont tendance à nous dire que rien ne nous empêche de leur fournir ces informations et c'est souvent vrai, mais rien ne nous y oblige non plus. (Entretien avec un Mlro, 2008)

D'un autre côté, les agents de police et de renseignement se sont longtemps retranchés derrière une vision de l'anti-blanchiment où la circulation d'informations devait être à sens unique, avec leurs services de sécurité comme points d'arrivée. La détention d'informations sensibles étant au fondement de leur pouvoir et de leur légitimité (Linhardt, 2005; Bigo, 2009; Gill \& Phythian, 2012), ces agents envisagent leur partage avec parcimonie en insistant sur la protection de leurs sources, de leurs méthodes et de leurs investigations en cours. Étant donné qu'il s'agit déjà d'un enjeu de lutte entre services étatiques, ils sont encore plus méfiants à l'idée de mutualiser leurs 
connaissances avec des agents d'institutions commerciales soumises à la législation anti-blanchiment.

Venant du renseignement, il y a une expression que nous utilisons régulièrement, celle du «besoin d'en connaître» (the need-to-know) qui signifie que l'on a besoin de connaître que les faits strictement nécessaires à la réalisation de notre tâche à un moment donné, rien de plus. Le secteur privé a parfois du mal à l'accepter et des agents de conformité nous reprochent de ne pas tout mettre sur la table. Il faut dire qu'il y a aussi le fantasme que nous en savons toujours plus que ce que nous laissons paraître. (Entretiens avec un représentant de l'Office of security and counter-terrorism, 2008)

Le principe du «besoin d'en connaître» sous-entend aussi que l'information «nécessaire» doit uniquement parvenir à quelques personnes clés et en aucun cas être diffusée au sein d'un réseau bancaire car, selon ce principe, l'information garde sa valeur si et seulement si son accès est protégé et limité.

La position des services de sécurité nous met dans une situation difficile car quand ils partagent des informations, ils nous demandent instamment de protéger ces informations en nous tenant responsables en cas de fuite, ce qui complique fortement nos capacités à les utiliser puisqu'on ne peut pas vraiment courir le risque de les distribuer en interne. (Entretien avec un Mlro, 2008)

Nonobstant les difficultés éprouvées et les critiques émises de part et d'autre, les professionnels britanniques de la surveillance financière parviennent tout de même à échanger des informations dans des conditions et des proportions présentées comme sans équivalent en Europe. Depuis 2006, plusieurs initiatives sont venues compléter les traditionnels coups de fil informels. Alors que des agents de conformité antiblanchiment ont été ponctuellement invités dans les murs de Scotland Yard, des agents de la Soca se sont régulièrement rendus au siège londonien des grandes institutions financières. Il s'agissait pour les premiers d'apporter leur expertise en matière de produits financiers et pour les seconds de contribuer à affiner les dispositifs bureaucratiques et technologiques de surveillance des établissements visités. Enfin, des zones de contact opérationnelles ont été institutionnalisées sous la forme de groupes de travail hybrides rassemblant des Mlros et des représentants de l'industrie financière avec des agents de la Soca et de l'unité d'investigation financière contre le terrorisme. La représentativité 
du monde financier est cependant limitée aux grandes banques et compagnies d'assurances couvrant 70 à $85 \%$ de la clientèle britannique, au grand dam des institutions de moindre envergure pour qui le «trafic» d'informations reste à sens unique. Il n'en reste pas moins que les heureux élus sont habilités à rencontrer des agents de l'État dans des environnements sécurisés.

Plusieurs groupes de travail fermés coexistent sous l'égide de la Soca dont le plus emblématique demeure le vetted group sur les déclarations de soupçon. Le rôle et le fonctionnement de ce dernier illustrent autant le dépassement de certaines réticences passées que l'ambiguiité du compromis actuel en matière de partage $\mathrm{d}^{\prime}$ «informations sensibles». Sa raison d'être est de favoriser l'échange et la discussion entre «partenaires» afin que les agents de conformité anti-blanchiment puissent mieux cibler leur surveillance en paramétrant leurs outils technologiques en fonction des indications confidentielles qui leur sont fournies (sur les outils de surveillance financière, voir Backhouse \& Canhoto, 2007; De Goede, 2008b; Amicelle \& Favarel-Garrigues, 2012). Pour ce faire, l'usage des données mises en commun doit respecter les règles de confidentialité qui s'appliquent à chacun. La plupart du temps, les discussions portent sur l'actualisation des tendances de blanchiment d'argent et de financement du terrorisme à partir de cas précis. Dans ce cadre, les agents de conformité sont susceptibles d'accéder à des renseignements portant sur des personnes et des entités mais, à quelques exceptions près, ils n'ont pas le droit de les mobiliser et de les diffuser en l'état sous peine d'être poursuivis pour violation de confidentialité. Pour remédier à cette situation, les membres du vetted group doivent donc mener une opération de désensibilisation des informations communiquées lors de leurs réunions. Ils s'attellent conjointement à transformer des pistes individuelles en typologies anonymisées servant à orienter la vigilance des équipes de gestion des risques au sein des institutions financières.

Le défi est d'accorder les exigences des forces de l'ordre et de la Soca en matière de création de typologies et de dissémination d'informations avec nos attentes afin d'arriver à un format utile et aisément manipulable dans nos banques. (Entretien avec un Mlro, 2008)

Tout l'art de cet exercice de traduction-généralisation consiste à trouver le point d'équilibre entre des renseignements d'État non diffusables et des typologies si banales qu'elles en perdent toute utilité opérationnelle. 
Je siège au vetted group de la Soca pour lequel il faut une accréditation. Oui, ils partagent de l'information avec vous, mais vous ne pouvez pas l'utiliser dans votre propre institution. L'objectif est bien sûr d'essayer d'affiner les profils de clients et d'activités à risque, mais si à la fin, c'est pour arriver à la conclusion que nous devrions regarder tous les dossiers avec des hommes aux cheveux noirs, ce n'est pas très pratique. (Entretien avec un Mlro, 2008)

\section{Un malentendu opératoire autour du risque d'u argent sale »}

\section{Risques «sociétaux» et risques «institutionnels»}

In fine, le vetted group joue avant tout un rôle de poste d'aiguillage dans la mesure où ses membres doivent déterminer les directions à emprunter pour les pratiques de surveillance financière. Malgré les frustrations exprimées ici et là, les professionnels rencontrés défendent l'utilité de ce type de groupe hybride qui leur permet de se rencontrer et de converser dans un langage qu'ils disent tous partager, celui de la gestion des risques. Défendue par les représentants du Royaume-Uni au Conseil et au Parlement européens ainsi que dans les fédérations bancaires à Bruxelles, cette grammaire du risque est promue dans les normes supranationales contre l'«argent sale» (Amicelle, 2011).

Bien sûr tout n'est pas parfait et il y aura toujours des choses à améliorer, mais au moins nous sommes tous tournés vers le même objectif qui est de gérer les risques de blanchiment d'argent et de financement du terrorisme, et c'est bien là l'essentiel, c'est ce qui nous permet d'avancer ensemble. (Entretien avec un Mlro, 2008)

Mis bout à bout, les entretiens que nous avons menés tendent à confirmer cette convergence linguistique entre deux «mondes», ceux de la finance et de la sécurité, dont les habitants semblent de prime abord partager une vision similaire de la lutte contre l'«argent sale». À l'ins$\operatorname{tar} \mathrm{du}$ vetted group, la surveillance financière dans son ensemble fonctionne à partir de cette terminologie du risque censée faciliter la communication entre des agents aux orientations différentes. Toutefois, si le langage du risque est effectivement usité chez les parties en présence, la pratique de ce nouvel esperanto reflète davantage une méprise sur le sens à donner au mot «risque» que le partage d'une représentation commune du risque à gérer. Les différences persistent dans les manières de faire et de penser l'anti-blanchiment. 
Certes, tous les professionnels de la surveillance financière s'accordent sur l'utilisation du même mot, mais ils ne parlent pas de la même chose. La juxtaposition des entretiens réalisés fait immédiatement apparaître un système de tensions entre finance et sécurité. Au regard de la mission qui leur est confiée, les agents de police et du renseignement appréhendent d'abord le risque d'«argent sale» comme une menace pour la population en l'associant à la violence criminelle et aux attentats. Les agents de la Soca se font ainsi les porte-parole de leur institution dont la mission est de «lutter contre le crime et garder la population en sécurité. En particulier, [il y a la volonté] de garantir que le crime ne paie pas ${ }^{9} . »$ En revanche, les agents de conformité antiblanchiment abordent avant tout le risque d'«argent sale» en termes de menace juridique, financière et réputationnelle pour eux, leurs employeurs et leur institution commerciale.

Si nous mettons en place tous ces mécanismes internes de gestion de risque dans nos banques, c'est pour deux raisons. Premièrement, nous faisons cela pour être en mesure d'attraper des criminels ou des terroristes, mais nous savons bien que cela reste très, très improbable. Deuxièmement, je vais être direct, mais la véritable raison pour laquelle nous le faisons, c'est pour éviter d'être poursuivi au Royaume-Uni ou aux États-Unis, voire de perdre notre licence ou d'avoir le régulateur financier nous disant que nous n'avons pas fait ce que nous aurions dû faire. (Entretien avec un Mlro, 2008)

Les uns parlent de risques «sociétaux» lorsque les autres ont en tête les risques «institutionnels» et les possibles conséquences négatives en cas de lien, même involontaire, avec de l'«argent sale». La convergence linguistique facilite peut-être le rapprochement entre professionnels de la surveillance financière, mais c'est au prix d'un malentendu autour du mot risque.

À cet égard, le malentendu opératoire autour du risque s'avère être la traduction particulière des diverses interprétations du «problème de l'argent sale» qui ont constitué le fondement ambivalent de la mobilisation internationale contre le blanchiment de capitaux.

La mobilisation anti-blanchiment: «une conjonction implicite d'intérêts»

«La lutte contre le blanchiment des capitaux ne revient pas seulement à lutter contre la criminalité, mais consiste aussi à préserver l'intégrité

9. Voir le site de la Soca: www.soca.gov.uk/about-soca 
des institutions financières et en définitive du système financier dans son intégralité» (citée dans Gilmore, 2005 : 102). Dans cette simple et courte phrase, Tom Sherman mobilise les deux registres discursifs ayant légitimé la mobilisation anti-blanchiment et l'existence du Groupe d'action financière (Gafi), dont il a été l'un des premiers présidents au début des années $1990^{10}$. D'un côté, une logique de chasse aux capitaux illicites a été promue pour orienter une partie des moyens policiers vers le «nerf de la guerre», l'argent (Sheptycki, 2000; Beare, 2003; Naylor, 2004). Visant à garantir que le «crime ne paie pas», l'idée d'attaquer les criminels au portefeuille a été actée internationalement dans la Convention des Nations Unies contre le trafic illicite de stupéfiants et de substances psychotropes en décembre 1988. D'un autre côté, une logique financière a été superposée à la stratégie policière et judiciaire au nom de la protection de l'intégrité et de la stabilité du système financier en empêchant l'utilisation des banques à des fins de blanchiment d'argent (Mitsilegas, 2003 ; Van Duyne, 2003 ; Reuter \& Truman, 2004; Hülsse, 2007). Également adoptée en décembre 1988, la déclaration du Comité de Bâle sur le contrôle bancaire précise que «la confiance du public dans les banques et, par conséquent, la stabilité de celles-ci peuvent être ébranlées par une publicité défavorable résultant d'une association involontaire des banques avec des criminels» (Comité de Bâle, 1988).

L'enchevêtrement de ces logiques policières et financières a été au fondement du Gafi et des normes internationales de 1990 censées apporter «une contribution décisive à la lutte contre les activités crimi-

10. Fondé en 1989 lors d'un sommet du G7 à Paris, le Groupe d'action financière (Gafi) est l'organisme intergouvernemental de référence chargé «de concevoir et de promouvoir des politiques de lutte contre le blanchiment de capitaux et le financement du terrorisme aussi bien à l'échelon national qu'international». Cela se traduit par la publication des 40 recommandations du Gafi. Ces normes internationales ont été révisées à plusieurs reprises depuis 1990. Le Gafi comprend aujourd'hui 36 membres, dont deux institutions régionales (la Commission européenne et le Conseil de coopération du Golfe) pour 34 pays et territoires. Huit formations «satellites» du Gafi s'ajoutent à ce premier cercle et regroupent chacune entre une demi-douzaine et une trentaine d'États-membres. Enfin, une vingtaine d'organisations, concernées de près ou de loin par la thématique de l'«argent sale», disposent d'un statut d'observateur au Gafi. Cela va de la Banque mondiale et du Fonds monétaire international à Interpol et Europol, en passant par le Comité contre le terrorisme du Conseil de sécurité des Nations Unies ou encore par le Comité de Bâle sur le contrôle bancaire. Au total, plus de 180 juridictions nationales sont formellement impliquées dans la lutte contre le blanchiment de capitaux et le financement du terrorisme dans la mesure où leurs représentants se sont engagés à appliquer les recommandations émanant du Groupe d'action financière. 
nelles et particulièrement contre le trafic de la drogue, et [permettre] de renforcer la solidité du système financier international» (Gafi, 1991: 21). Loin d'être l'exclusive de l'une ou de l'autre, la lutte contre l'«argent sale» est bien située à l'interface de ces deux logiques d'action. Les premières réunions du Gafi ont accueilli des centaines «d'experts de différents ministères, autorités de détection et de répression, et organes de contrôle et de réglementation bancaire [...] qui ont travaillé ensemble pendant six mois» (Gafi, 1991: 4). Selon Bernard Gravet, les convergences nationales en matière anti-blanchiment refléteraient une «conjonction implicite d'intérêts» entre des groupes d'acteurs issus des ministères de l'Intérieur et de la Justice et des groupes d'acteurs issus des ministères des Finances et des autorités de supervision bancaire ${ }^{11}$ (Gravet \& Favarel-Garrigues, 2003). Toujours est-il que l'émergence des normes internationales découle en grande partie de ce rapprochement entre différents positionnements bureaucratiques qui affleurent à la surface des positions nationales.

De cela transparait l'intentionnalité non univoque au fondement du consensus contre l'«argent sale», dans lequel coexistent et doivent s'ajuster en permanence deux objectifs qui, sans être nécessairement antagonistes, sont différents, à savoir celui de protéger l'intégrité et la stabilité de l'ordre financier existant et celui de lutter contre la criminalité. Structurée autour de cette dualité finance/sécurité, la configuration anti-blanchiment est ainsi formée par un ensemble de tensions entre différents acteurs ou joueurs, pour reprendre la métaphore du jeu chère à Norbert Elias. «L'interdépendance des joueurs, condition nécessaire à l'existence d'une configuration spécifique, est une interdépendance en tant qu'alliés, mais aussi en tant qu'adversaires» (Elias, 1991: 157). Dans ce système de tensions entre deux grands groupes d'acteurs en situation d'interdépendance, l'enjeu a été pour chacun d'affirmer, voire d'imposer sa vision légitime de ce que doit être la lutte contre l'«argent sale» (Amicelle, sous presse).

La mobilisation britannique résulte également de cette combinatoire d'intérêts ou plutôt de cet équilibre de tensions entre finance et sécurité. D'un côté, le Trésor est officiellement responsable de la gestion de la «menace» représentée par le blanchiment de capitaux pour le système financier britannique et s'occupe du cadre réglementaire pour combattre

11. Ancien directeur central de la police judiciaire française, Bernard Gravet a participé au développement des mécanismes anti-blanchiment en France et au sein du Gafi. 
la finance illicite, y compris le financement du terrorisme et de la prolifération nucléaire (HM Treasury, 2012). Cet effort de protection de l'industrie financière britannique est mené conjointement avec l'autorité indépendante de supervision financière (Financial Services Authority - FSA). De l'autre, le Home Office couvre les aspects ayant trait à la coopération policière et judiciaire et aux pratiques de renseignement antiterroriste. Ce ministère de l'Intérieur comprend depuis 2007 un bureau pour la sécurité et le contre-terrorisme (Office for Security and Counter-Terrorism) dont l'objectif principal est de protéger le public en matière de terrorisme; ses agents coordonnent la stratégie britannique contre le terrorisme, y compris son volet financier dont ils sont responsables avec leurs homologues du Trésor. La Soca est également sous la tutelle du Home Office.

Le Trésor et le secteur bancaire ont une expertise en matière de services financiers et en matière de standards internationaux avec le Gafi, alors que nous, nous voyons davantage le cœur de notre expertise du côté de la criminalité et du contre-terrorisme au sens large. Il y a donc différents ministères et différents secteurs avec différentes sortes d'expertise et on ne peut pas divorcer les deux. (Entretiens avec un représentant de l'Office for security and counter-terrorism, 2008)

Cette répartition des tâches entre les deux ministères et les justifications qui l'accompagnent témoignent encore une fois des deux logiques d'action structurant le champ d'intervention anti-blanchiment, l'une financière et l'autre policière. La fonction de Money Laundering Reporting Officer résulte de ce mariage de raison et en cristallise toutes les tensions.

\section{Les effets controversés de la surveillance financière}

Un système de tensions intériorisé

Par leur simple existence, les Money Laundering Reporting Officers matérialisent l'articulation entre finance et sécurité dans toute sa complexité puisque le système de tensions ne se joue pas seulement entre agences policières et bancaires, il traverse également les institutions financières de part en part.

La finalité d'une banque est de faire de l'argent, la finalité d'une banque n'est pas de faire un travail policier, mais il faut bien reconnaitre que nous 
jouons presque le rôle d'une police interne au sein de l'institution financière, ce qui peut parfois susciter des tensions avec nos services commerciaux. (Entretien avec un Mlro, 2008)

Selon les dispositions sociales à partir desquelles ils perçoivent et accomplissent leur mission, les agents de conformité anti-blanchiment peuvent eux-mêmes être tiraillés à titre individuel par ce système de tensions. La faible épaisseur historique de leur fonction fait que les pionniers des années 1990, qui occupent actuellement les plus hautes responsabilités, ont été professionnellement socialisés dans d'autres services. Une partie de ces pionniers est issue $\mathrm{du}$ «monde» financier. Ils ont été recrutés en interne, notamment dans les rangs des unités de conformité et des services antifraude où certains ont pu faire valoir leur formation juridique. L'autre partie est issue du «monde» de la sécurité, à savoir des agents de police et de renseignement enrôlés pour leur carnet d'adresses et leur capacité à obtenir des informations utiles ${ }^{12}$. Selon leur trajectoire, ils se sont familiarisés aux problématiques financières dans les années 1980 et 1990 en enquêtant sur des affaires de fraudes ou de corruption, de trafic de stupéfiants ou bien encore sur des cas de violence politique et sur des organisations désignées «terroristes». Leur façon de se tenir et de s'exprimer, en un mot leur hexis corporel (Bourdieu, 1980), trahit l'importance de leur première carrière dans leur manière de penser et d'agir en tant que Mlro. Ces anciens agents de l'État ont précisément été recrutés pour ces «dispositions mentales et comportementales professionnellement constituées» (Lahire, 2012: 47), mais ce passé incorporé rend d'autant plus saillante la tension entre finance et sécurité.

Vous savez, je travaille pour trop de personnes. Je travaille pour mon Étatmajor, je travaille pour mes actionnaires, je travaille pour les forces de l'ordre, je travaille pour le régulateur financier, je travaille pour le gouvernement, je travaille pour moi [...]. Je suis payé par ma direction qui veut de moi que je fasse mon travail aussi efficacement que possible, c'est-à-dire pour un coût minimum permettant aux affaires de crô̂tre autant que possible, ce qui peut m'exposer à des situations et des décisions difficiles. La situation est plus critique en période de crise financière où les agents commerciaux se font plus pressants en cherchant l'argent là où ils n'auraient même pas regardé un an auparavant. Ensuite vous avez les forces de l'ordre qui poussent pour obtenir le plus d'informations possible et le plus rapi-

12. Il n'y a là rien de spécifique au Royaume-Uni (Favarel-Garrigues et al., 2008). 
dement possible, c'est-à-dire souvent en off, en dehors des procédures officielles. Puis vous avez la FSA [régulateur financier] qui veut au contraire que je documente et que je justifie par écrit tout ce que je fais. Et finalement, il y a moi [...]. (Entretien avec un Mlro, 2008)

Il arrive souvent que ces agents ne soient pas en mesure d'obtenir autant d'informations qu'ils le souhaiteraient en interne, surtout quand ils travaillent pour un établissement dont le siège n'est pas au RoyaumeUni. Ils sont alors amenés à gérer en permanence la tension entre l'ambition de contribuer à lutter contre la délinquance financière et les contraintes instituées au sein de leur institution commerciale. Cette tension est appréhendée différemment selon la trajectoire professionnelle des Mlros.

Toutefois, si le fait d'exercer la fonction d'agent de conformité antiblanchiment réactive des habitudes et mobilise des compétences déjà constituées, cela favorise également le développement d'autres manières de voir et d'agir, y compris chez d'anciens policiers qui n'hésitent pas à se présenter comme des banquiers. En tant que contextes d'actions, les banques et leurs directions de gestion des risques sont simultanément des «cadres déclencheurs de dispositions incorporées» et des «cadres socialisateurs» pour ces agents de l'État reconvertis dans la sphère commerciale (Lahire, 2012: 26). Quelles que soient leur socialisation professionnelle et leur propension à aborder la question de l'«argent sale», les Mlros s'accordent sur la finalité première de leur métier qui n'est pas de faire baisser le taux de criminalité au RoyaumeUni. Leur mission consiste avant tout à protéger leur institution financière et leur état-major contre tout risque juridique et réputationnel inhérent à l'«argent sale». C'est dans cette perspective qu'ils sont enclins à partager toujours plus de données avec les agents des services de police et de renseignement, en particulier sur les enjeux de financement du terrorisme. Néanmoins, les efforts qu'ils entreprennent dans la gestion des risques «institutionnels» vont parfois à l'encontre des attentes exprimées pour gérer les risques «sociétaux» (Ericson, 2006; Rothstein et al. 2006; Power, 2007; Hood, 2011).

\section{Fichiers de suspects et droits fondamentaux}

Agissant dans un contexte de défense organisationnelle, nombre d'agents de conformité anti-blanchiment effectuent des «déclarations de complaisance» afin d'éviter toute mise en cause future. Autrement 
dit, ils ont tendance à interpréter le moindre doute en soupçon suffisant pour signaler toute transaction «inhabituelle» à la cellule de renseignement financier. Le doute prime au nom d'une aversion au risque institutionnel même si cela a pour conséquence de sur-déclarer aux services de sécurité en produisant plus de bruit que de renseignement éventuellement utile à la gestion des risques sociétaux. Si ces stratégies d'évitement de responsabilité visent à protéger les institutions financières, c'est au détriment de l'autre objectif affiché de la mobilisation contre l'«argent sale», à savoir la lutte contre la criminalité. Le malentendu opératoire autour de la notion de risque oscille alors entre conjonction et disjonction implicite d'intérêts.

Au rythme annuel de 200000 déclarations de soupçon à traiter, les agents de la Soca estiment qu' ils reçoivent «une proportion significative de déclarations purement défensives» tout en se disant incapables d'en évaluer l'ampleur avec précision (Entretien avec deux agents de la Soca, 2008). Ils sont donc parfaitement conscients des effets inattendus produits par le malentendu fondateur des possibilités de surveillance et de contrôle des flux financiers illicites. Toutefois, ils s'en accommodent en des termes qui entrent directement en résonance avec la croyance contemporaine faisant du toujours plus de données la solution aux problèmes de sécurité présentés comme fluides, transnationaux et imprévisibles (Leman-Langlois, 2011 ; Amoore \& De Goede, 2012).

Il y a beaucoup de signalements défensifs mais quand on regarde la façon dont on utilise notre base de données [regroupant les déclarations] pour rechercher et faire de l'exploration de données, quand on regarde cela d'un peu plus près, ce qui apparaît aujourd'hui comme une déclaration défensive pourrait être dans deux ans un rapport contextuel efficace pour aider les agences d'investigation et de renseignement ${ }^{13}$. (Entretien avec deux agents de la Soca, 2008)

Cependant, ce type de raisonnement a été récemment critiqué par les agents de l'autorité britannique en matière de protection des données personnelles (Information Commissioner). Ces derniers ont remis en cause

13. L'exploration de données ou le data mining est la procédure par laquelle de larges bases de données sont analysées au moyen d'algorithmes relatifs à des modèles de corrélations entre données (Gutwirth \& Hildebrandt, 2008). Il s'agit souvent d'interpréter une base de données, de dégager un modèle explicatif permettant de décrire ou de résumer les informations qu'elle contient, de trouver ce qui la sous-tend, en dévoilant l'«invisible», en faisant ressortir des relations ou des similitudes existant entre les données mais qui étaient restées masquées jusqu'ici. 
l'un des principaux résultats de la surveillance financière au RoyaumeUni, à savoir la production et le maintien d'un imposant fichier de personnes suspectes. À l'instar de leurs corrélats européens, les agents de la Soca ont en effet pour mission de centraliser les déclarations de soupçon des institutions financières dans un fichier national et de les conserver pour une durée maximum de dix ans. En indiquant le montant, l'origine et la destination présumés des fonds suspects, les déclarants identifient de facto des personnes suspectes et fournissent toute une série d'informations les concernant. Chaque fiche doit ainsi comprendre le nom complet de l'individu visé, sa date et son lieu de naissance, sa nationalité, son ou ses adresses, son numéro et son type de compte bancaire, des précisions sur sa profession, d'autres précisions inscrites sur son passeport et son permis de conduire, l'immatriculation de son véhicule, ses coordonnées téléphoniques et électroniques, ainsi que des éléments relatifs à sa «surface financière connue», c'est-à-dire les revenus issus de son occupation professionnelle, ses emprunts en cours ou encore l'étendue de son patrimoine. Le fichier central de la Soca a compté jusqu'à un million neuf cent mille entrées ces dernières années, ce qui en fait le plus volumineux de l'Union européenne (House of Lords, 2011).

Dans un rapport rendu public en 2011, les agents de l'Information Commissioner ont mis en question le mode de production et de gestion d'un tel fichier. D'une part, ils ont considéré que par son ampleur, «la rétention de données [dans ce fichier] soulève des préoccupations sur le fait de savoir s'il s'agit d'une interférence injustifiée avec le droit des individus au respect de leur vie privée et familiale» (Information Commissioner, 2011 : 19). D'autre part, ils ont abordé la problématique des déclarations de complaisance massivement enregistrées dans le fichier en demandant s'il existait «un besoin social impérieux justifiant la nécessité de signaler toute transaction sur la base d'un seuil de soupçon très bas pouvant indiquer la présence de biens criminels ou de blanchiment de capitaux» (Information Commissioner, 2011 : 20). À la suite de ce rapport et des débats qu'il a suscités, les représentants de la cellule britannique de renseignement financier ont été amenés à effacer 584351 déclarations de soupçon dont la conservation était jugée infondée (Bamford, 2012). Le monde de la surveillance financière est caractérisé par une tension fondamentale à l'interface de la finance et de la sécurité, mais d'autres points de tension surgissent également au contact d'acteurs défenseurs de droits fondamentaux. 


\section{Conclusion}

Contredisant les analyses en termes d'engagement idéaliste (Finnemore \& Sikkink, 1998), la lutte contre l'«argent sale» renvoie avant tout à un mariage de raison dans lequel les intérêts des deux groupes d'acteurs en présence priment sur le partage d'une vision commune. La surveillance financière telle qu'elle est définie dans la configuration financesécurité est porteuse de plusieurs visions précises de l'enjeu traité. Loin d'être un obstacle rédhibitoire, l'absence de définition univoque du «problème» et des «solutions» est paradoxalement ce qui a permis de mobiliser aussi largement contre le blanchiment de capitaux et le financement du terrorisme. En effet, les divergences d'interprétations et les méprises sur le sens des mots, autrement dit les malentendus, sont précisément les éléments qui font marcher bon an mal an le monde de la surveillance financière.

«L'enrôlement des intérêts professionnels autour d'un projet reste la meilleure garantie de son bon accomplissement» (Aust \& Purenne, 2010 : 24). Souvent associée à la mise en œuvre d'une politique, cette remarque vaut aussi pour sa mise en forme juridique. La notion de «bon accomplissement» est ici à prendre dans un sens bien précis qui s'éloigne «des formulations globales et univoques en termes de "succès" ou d'“échecs"» (Bezes, 2009: 7). Il s'agit simplement de noter que le projet de lutte contre l'«argent sale» n'est pas resté lettre morte. Il est en cela effectif puisqu'il produit bel et bien des effets en engendrant des pratiques inédites de surveillance et d'échange d'informations qui mettent à l'épreuve les frontières institutionnelles de l'État vis-à-vis de ses missions de police et de renseignement. De là à en conclure que cette politique de compromis et d'ajustement permanent entre des initiatives de protection financière et de lutte contre la criminalité est efficace au-delà d'être effective, c'est une question qui demeure ouverte et sujette à de vifs débats qui dépassent le cercle des praticiens de l'antiblanchiment. Si l'articulation fragile de plusieurs visions d'une même politique permet de maintenir un large consensus autour de malentendus souvent bien entendus, l'ambivalence qui s'en dégage peut produire des effets controversés, en particulier lorsque cette politique est susceptible d'affecter la dynamique des relations entre surveillance, sécurité et liberté. 


\section{Références}

Amicelle, A. (2008). La lutte contre le financement du terrorisme. In D. Bigo, L. Bonelli \& T. Deltombe (eds.), Au nom du 11 septembre... Les démocraties à l'épreuve de l'antiterrorisme (131-138). Paris: La Découverte.

Amicelle, A. (2011). Towards a 'new' political anatomy of financial surveillance. Security dialogue, 42 (2), 161-178.

Amicelle, A. (2012). Trace my money if you can: European security management of financial flows. In U. Morth \& K. S. Helgesson (eds.), Securitization, Accountability and Risk Management: Transforming the Public Security Domain, (110-131). London: Routledge.

Amicelle, A. (2013). Gestion différentielle des illégalismes économiques et financiers. Champ pénal/Penal Field, 10. Consulté le 14 juin 2013, http:// champpenal.revues.org/8403

Amicelle, A. (sous presse). Entre sécurité nationale et sécurité financière: retour sur la construction sociale de l'argent sale. In J. Neves Cruz, C. Cardoso, A. Leite Lamas \& R. Faria (eds.), Economic and Financial Offenses: Criminology $\mathfrak{F}$ Law Studies. Porto: Coimbra Editora, Wolters Kluwer Portugal.

Amicelle, A., \& Favarel-Garrigues, G. (2012). Financial surveillance: Who cares? Journal of Cultural Economy, 5 (1), 105-124.

Amoore, L., \& De Goede, M. (2012). Introduction: Data and the war by other means. Journal of Cultural Economy, 5 (1), 3-8.

Aust, J., \& Purenne, A. (2010). Piloter la police par les indicateurs? Effets et limites des instruments de mesure des performances. Déviance et Société, 34, 7-28.

Backhouse, J., \& Canhoto, A. (2007). Profiling under conditions of ambiguity: An application in the financial services industry. Journal of Retailing and Consumer Services, 14 (6), 408-419.

Bamford, J. (2012). Privacy and data protection: Are they casualties in the fight against crime? London: Information Commissioner's Office.

Beare, M. E. (Ed.). (2003). Critical reflections on transnational organized crime, money laundering, and corruption. Toronto: University of Toronto Press.

Bezes, P. (2009). Réinventer l'État. Paris: Presses universitaires de France.

Bigo, D. (2006). Globalized-in-security: The field and the ban-opticon. In N. Sakai et J. Solomon (eds.), Translation, Biopolitics, Colonial Difference (109156). Hong Kong: University of Hong Kong Press.

Bigo, D. (2009). Un espace de liberté, de sécurité et de justice? In R. Dehousse (ed.), Politiques européennes (331-352). Paris: Presses de Sciences Po.

Bourdieu, P. (1980). Le sens pratique. Paris : Éditions de minuit.

Comité de Bâle. (1988). Prévention de l'utilisation du système bancaire pour le blanchiment de fonds d'origine criminelle. Bâle: Comité de Bâle.

Commission européenne. (2008). Study on "Best practices in vertical relations between the Financial Intelligence Unit and (1) law enforcement services and (2) Money Laundering and Terrorist Financing Reporting entities with a view to indicating effective models for deedback on follow-up to and effectiveness of suspicious transaction reports". Brussels: B\&S Europe. 
Conseil de l'Union européenne. (2008). Stratégie révisée de lutte contre le financement du terrorisme. Bruxelles: Conseil de l'Union européenne.

De Goede, M. (2008a). The politics of preemption and the war on terror in Europe. European Journal of International Relations, 14 (1), 161-185.

De Goede, M. (2008b). Risk, preemption and exception in the war on terrorist financing. In L. Amoore et M. De Goede (eds.), Risk and the War on Terror (97-112). London: Routledge.

De Goede, M. (2012). Speculative security: The politics of pursuing terrorist monies. Minneapolis: University of Minnesota Press.

Dezalay, Y., \& Rask Madsen, M. (2006). La construction européenne au carrefour du national et de l'international. In A. Cohen, B. Lacroix \& P. Riutor (eds.), Les formes de l'activité politique. Éléments d'analyse sociologique XVIII ${ }^{e}-$ $X X^{e}$ siècle (277-296). Paris: PUF.

Dupuis-Danon, M.-C. (2004). La lutte contre la finance criminelle: financement du terrorisme et blanchiment d'argent sale. Géoéconomie, 31, 63-76.

Elias, N. (1991). Qúest-ce que la sociologie. Paris: Éditions de l'Aube.

Ericson, R. (2006). Ten uncertainties of risk-management: Approaches to security. Canadian Journal of Criminology and Criminal Justice, 48 (3), 345-357.

Ericson, R. (2008). The state of preemption: Managing terrorism risk through counter-law. In L. Amoore \& M. De Goede (eds.), Risk and the War on Terror (57-76). London: Routledge.

Favarel-Garrigues, G., Godefroy, T., \& Lascoumes, P. (2008). Sentinels in the banking industry. The British Journal of Criminology, 48 (1), 1-19.

Favarel-Garrigues, G., Godefroy, T., \& Lascoumes, P. (2009). Les sentinelles de l'argent sale: les banques aux prises avec l'antiblanchiment. Paris: La Découverte.

Fines, L. (2004). Le financement d'activités terroristes au Canada: collecte, blanchiment, transfert et cadre juridique. ERTA-TCRG. Consulté le 14 juin 2013, www.erta-tcrg.org/analyses/financement.htm

Finnemore, M., \& Sikkink, K. (1998). International norms, dynamics and political change. International Organization, 42 (4), 887-917.

Gill, P., \& Phythian, M. (2012). Intelligence in an insecure world. Cambridge: Polity Press.

Gilmore, W. (2005). L'argent sale: l'évolution des mesures internationales de lutte contre le blanchiment des capitaux et le financement du terrorisme. Strasbourg: Éditions du Conseil de l'Europe.

Gravet, B., \& Favarel-Garrigues, G. (2003). De l'argent de la drogue à l'argent sale: l'invention de la lutte anti-blanchiment (entretien avec Bernard Gravet; propos recueillis par Gilles Favarel-Garrigues). Cabiers de la sécurité intérieure, 52, 141-158.

Groupe d'action financière (Gafi). (1991). Rapport annuel. Paris: Groupe d'action financière sur le blanchiment de capitaux.

Gutwirth, S., \& Hildebrandt, M. (Eds.). (2008). Profiling the European citizen: Cross-disciplinary perspectives. Dordrecht: Springer.

Haggerty, K., \& Razavy, M. (2009). Hawala under scrutiny: Documentation, surveillance and trust. International Political Sociology, 3 (2), 139-155. 
Hayes, B., \& Sullivan, G. (2010). Blacklisted: Targeted sanctions, preemptive security and fundamental rights. Berlin: European Center for Constitutional and Human Rights; 10 years after 9/11 Publication Series.

Hibou, B. (2011). Anatomie politique de la domination. Paris: La Découverte.

HM Treasury. (2007). The financial challenge to crime and terrorism. London: HM Treasury.

HM Treasury. (2012). UK anti-money laundering $\mathfrak{O}$ counter terrorist finance policy. London: HM Treasury.

Hood, C. (2011). The blame game. Spin, bureaucracy and self-preservation in government. Princeton: Princeton University Press.

Hood, C., Rothstein, H., \& Baldwin, R. (2001). The Government of risk: Understanding risk regulation regimes. Oxford: Oxford University Press.

House of Lords. European Union Committee. (2011). Money Laundering: Data protection for suspicious activity reports. London: United Kingdom Parliament.

Hülsse, R. (2007). Creating demand for global governance: The making of a global money-laundering problem. Global Society, 21 (2), 155-178.

Information Commissioner. (2011). The Serious Organised Crime Agency's operation and use of the ELMER database. London: United Kingdom Parliament.

Lahire, B. (2012). Monde pluriel. Penser l'unité des sciences sociales. Paris: Éditions du Seuil.

Lascoumes, P. (1986). Les affaires ou l'art de l'ombre: les délinquances économiques et financières et leur contrôle. Paris: Centurion.

Leman-Langlois, S. (Ed.). (2011). Sphères de surveillance. Montréal: Les Presses de l'Université de Montréal.

Levey, S. (2006). Testimony of Stuart Levey, Under Secretary Terrorism and Financial Intelligence U.S. Washington: U.S. Treasury Department, Office of Public Affairs.

Levi, M. (2010). Combating the financing of terrorism. A history and assessment of the control of "threat finance". British Journal of Criminology, 50 (4), 650-669.

Linhardt, D. (2005). La «question informationnelle». Éléments pour une sociologie politique des fichiers de police et de population en Allemagne et en France. Déviance et Société, 29, 259-272.

McCulloch, J., \& Pickering, S. (2009). Pre-crime and counter-terrorism: Imagining future crime in the "War on Terror". British Journal of Criminology, 49 (5), 628-645.

Mitsilegas, V. (2003). Countering the chameleon threat of dirty money: "Hard" and "Soft" law in the emergence of a global regime against money laundering and terrorist finance. In A. Edward \& P. Gill (eds.), Transnational Organised Crime: Perspectives on Global Security (195-211). London; New York: Routledge.

Munzele Maimbo, S., \& Passas, N. (2008). The design, development, and implementation of regulatory and supervisory frameworks for informal funds transfer systems. In T. Biersteker \& S. Eckert (eds.), Countering the Financing of Terrorism (174-192). New York: Routledge. 
Naylor, R. (2004). Wages of crime: Black markets, illegal finance, and the underworld economy (Revised ed.). Ithaca; London: Cornell University Press.

Power, M. (2004). The risk management of everything: Rethinking the politics of uncertainty. London: Demos.

Power, M. (2007). Organized uncertainty: Designing a world of risk management. Oxford: Oxford University Press.

Reuter, P., \& Truman, E. (2004). Chasing dirty money: The fight against money laundering. Washington D. C.: Institute for International Economics.

Rothstein, H., Huber, M., \& Gaskell, G. (2006). A theory of risk colonization: The spiralling regulatory logics of societal and institutional risk. Economy and Society, 35 (1), 91-112.

Serious Organised Crime Agency (SOCA). (2012a). SARs Regime Annual Report 2012. London: SOCA.

Serious Organised Crime Agency (SOCA). (2012b). What is the UK Financial Intelligence Unit? London: SOCA.

Sheptycki, J. (2000). Policing the virtual launderette: Money laundering and global governance. In J. Sheptycki (ed.), Issues in Transnational Policing (135176). London; New York: Routledge.

Traitement du Renseignement et Action Contre les Circuits Financiers Clandestins (Tracfin). (2012). Rapport annuel 2011. Paris.

Union européenne. (1991). Directive 91/308/CEE du Conseil, du 10 juin 1991, relative à la prévention de l'utilisation du système financier aux fins du blanchiment de capitaux. Journal officiel, L 166. Consulté le 16 juin 2013, http://europa. eu/legislation_summaries/other/124016_fr.htm

Union européenne. (2005). Directive 2005/60/CE du Parlement européen et du Conseil du 26 octobre 2005 relative à la prévention de l'utilisation du système financier aux fins du blanchiment de capitaux et du financement du terrorisme. Journal officiel de l'Union européenne n L 309. Consulté le 16 juin 2013, http://europa.eu/legislation_summaries/justice_freedom_security/fight_ against_organised_crime/124016a_fr.htm

Van Duyne, P. (2003). Money laundering policy: Fears and facts. In P. Van Duyne, K. Von Lampe \& J. Newell (eds.), Criminal finances and organising crime in Europe (72-109). Nijmegen: Wolf Legal Publishers.

Vlcek, W. (2006). Acts to combat the financing of terrorism: Common Foreign Security Policy at the European Court of Justice. European Foreign Affairs Review, 11 (4), 491-507.

ABSTRACT - "Follow the money" is now a well-established practice of policing and intelligence. The fight against money laundering and terrorist financing is located at the interface of finance and security. Financial surveillance is based on cooperation between two groups of actors who have different motivations and different know-how. Indeed, the blurred notion of "dirty money" has become the meeting point of a constellation of interests and of two logics of action that are related to law enforcement authorities and financial institutions. With respect to the anti-money laundering apparatus in United Kingdom, the article highlights the articulation of these logics of 
action around a misunderstanding regarding the meaning of the fight against "dirty money". Here, the misunderstanding does not represent an obstacle to the elaboration and the operationalization of a specific policy. On the contrary, it is a precondition for this policy. While there are oversights and differences of interpretation about the "problem" to be addressed and the aims to be achieved, this situation paradoxically contributes to the formation of the mixed battalion of financial surveillance professionals.

KEYWORDS - dirty money, misapprehension, intelligence, risk, surveillance.

RESUMEN - El seguimiento de la pista financiera forma parte, hoy en día, de las prácticas instituidas en materia policial y de inteligencia. Situado en el interfaz entre las finanzas y la seguridad, la lucha contra el blanqueo de capitales y el financiamiento del terrorismo reposa sobre las relaciones de cooperación tejidas entre dos grupos de actores con motivaciones y competencias diferentes. La nebulosa noción de "dinero sucio " es, en efecto, el punto de encuentro de una constelación de intereses y de dos lógicas de acción propias a las fuerzas del orden y a los operadores del sistema financiero. Analizando el dispositivo antiblanqueo del Reino Unido, el presente artículo saca a relucir la frágil articulación entre dichas lógicas de acción en torno al malentendido existente en cuanto al sentido de la lucha contra el "dinero sucio". Lejos de constituir un obstáculo para la elaboración y la operación de una política específica, el malentendido representa aquí la condición misma de posibilidad. Desconsideraciones y divergencias de interpretación sobre el "problema" a tratar y los objetivos a seguir contribuyen, paradójicamente, a la formación de un abigarrado batallón de profesionales de la vigilancia financiera.

PALABRAS CLAVE - dinero sucio, malentendido, inteligencia, riesgo, vigilancia. 